\title{
The roles of convergence and apparent distance in depth constancy with motion parallax
}

\author{
JOSÉE RIVEST and HIROSHI ONO \\ York University, North York, Ontario, Canada \\ and \\ SHINYA SAIDA \\ Industrial Products Research Institute, Tsukuba City, Ibaraki, Japan
}

\begin{abstract}
The question of whether motion parallax is calibrated by convergence or by apparent distance for depth perception was addressed in three experiments. In Experiment 1, a random dot parallactic display was viewed monocularly at a distance of $80 \mathrm{~cm}$, and the convergence angles were set for distances of 40,60 , and $80 \mathrm{~cm}$. Averaged apparent depth was not different across conditions. In Experiment 2, a display consisting of one surface showing dollar bills and one surface showing random dots was viewed monocularly at a distance of $80 \mathrm{~cm}$. It was presented at two different apparent distances, which were manipulated by varying the size of the dollar bills. In one condition, normally sized dollar bills were presented, and in another condition, the size was reduced by $30 \%$. The averaged apparent depth associated with the small-bill display was larger than the depth associated with the normally sized bill display. In Experiment 3, a random dot display was viewed monocularly at $120 \mathrm{~cm}$. In the primary condition, the random dot display was viewed with an induction screen at $80 \mathrm{~cm}$, and it was moved from side to side such that it appeared stationary and close to the plane of the induction screen. In a comparison condition, the display was viewed without the induction screen and was moving from side to side at $120 \mathrm{~cm}$. In another comparison condition, the display was again viewed without the induction screen but was stationary at $120 \mathrm{~cm}$. Observers adjusted the extent of motion parallax so that apparent depth was $1 \mathrm{~cm}$. The mean extent of parallax was larger in the primary condition. The results show that the visual system calibrates motion parallax with apparent distance, but not directly with an oculomotor adjustment of convergence.
\end{abstract}

Since the turn of this century, motion parallax produced by head movement has been accepted as an important cue for depth perception (see Bourdon, 1902; Heine, 1905; Helmholtz, 1910/1962). According to Bourdon (1902), motion parallax is the principle cue for depth perception under monocular viewing. In recent years, interest in motion parallax has revived (see Graham, 1983; Nakayama, 1985; and Rogers \& Graham, 1979, for recent reviews), and the question of depth constancy as resulting from motion parallax has been explicitly raised (M. E. Ono, Rivest, \& H. L. Ono, 1986; Rogers, 1986).

This research was supported by Grant A0296 from the Natural Sciences and Engineering Research Council of Canada, Grant EY05960 from the National Institute of Health, and a grant from the International Program for Research Co-operation of Science and Technology, Japan. Experiments 1 and 2 were part of Josee Rivest's MA thesis and were presented at the Association of Research in Vision and Ophthalmology's meeting in 1987. The authors wish to thank R. Bird, P. Cavanagh, R. Comtois, D. Giaschi, W. Gogel, I. P. Howard, A. Mapp, and M. J. Steinbach for helpful comments on an earlier version of this article. They also wish to thank the two anonymous reviewers and $M$. Braunstein for comments on the originally submitted manuscript. Correspondence concerning this article should be addressed to Jose Rivest, who is now in the Department of Psychology, Harvard University, William James Hall, 33 Kirkland St., Cambridge, MA 02138.
To obtain depth constancy from motion parallax, the visual system must calibrate motion parallax information with absolute distance information. This calibration is necessary, because the extent of motion parallax changes as viewing distance changes. For example, if one looks at a three-dimensional object from one distance and then looks at the same object from a distance twice as great, the extent of motion parallax will be reduced to one fourth, but the apparent depth within the object will remain the same (Ittelson, 1960; M. E. Ono et al., 1986). Therefore, one of the essential conditions for depth constancy is that information about absolute or egocentric distance must be processed accurately: The visual system must process the scalar absolute distance by using absolute distance cues such as accommodation, convergence, vertical angle of regard, familiar size cues (see H. Ono, 1970), and/or a combination of relative distance cues and additional information such as the observer's translational velocity (see Nakayama, 1985). ${ }^{1}$

Although the use of absolute distance information with motion parallax has been implicated in depth constancy only recently, the role of retinal disparity has been studied for some time (see H. Ono \& Comerford, 1977, for a review of earlier work). Several authors have shown that depth constancy is maintained as retinal disparity changes 
with viewing distance (e.g., Fried, 1973/1974; Wallach \& Zuckerman, 1963), and other authors have examined the sources of absolute distance information used to calibrate retinal disparity (e.g., Cormack \& Menendez, 1983; Fried, 1974; Gogel, 1972, 1978; O'Leary \& Wallach, 1980; Wallach, Gillam, \& Cardillo, 1979; Wallach \& Zuckerman, 1963). The literature shows that the visual system calibrates binocular disparity with ocular motor adjustments, including accommodation and convergence (e.g., Wallach \& Zuckerman, 1963), and with apparent distance, as manipulated by the retinal size of familiar objects and by linear perspective (e.g., O'Leary \& Wallach, 1980).

In contrast, what absolute distance information is used to calibrate motion parallax has never been studied. Therefore, in the present study, the role of convergence is investigated in Experiment 1, and the role of apparent distance is investigated in Experiments 2 and 3.

\section{EXPERIMENT 1}

Experiment 1 was designed to determine whether apparent depth produced by motion parallax varies with the convergence state. Observers were asked to report the apparent depth arising from a random dot parallactic display presented at one distance under the following conditions: normal convergence-convergence angle set for the actual distance of the display $(80-\mathrm{cm}$ convergence condition); overconvergence - convergence angles set for three quarters of the actual distance $(60-\mathrm{cm}$ convergence condition); and again overconvergence-convergence angles set for half the actual distance $(40-\mathrm{cm}$ convergence condition). If apparent depth varies with the convergence state, then it should be smaller in the overconvergence conditions than in the normal convergence condition.

\section{Method}

The display used in Experiment 1 was identical to that used in the study by M. E. Ono et al. (1986). It simulated a sinusoidally corrugated three-dimensional surface. A video camera (Sony AVC3200) displayed "random dots" (a matrix with half the cells filled in) on a TV monitor. The observer's head was supported on a chinrest that could move $30 \mathrm{~cm}$ along a track parallel to the front of the monitor. A potentiometer was used to yoke the horizontal movement of the observer's head to the movement of the dots on the screen: The amplitude of the horizontal deflection signal of the monitor was modified by a sinusoidal signal from a function generator (Wavetek Model 184) in synchrony with the vertical signal of the monitor. As the head moved, horizontal bands of dots (or cells) representing the troughs of the corrugations moved across the screen in the same direction as the observer's head movement, while those representing the peaks of the corrugations moved in the opposite direction. (See M. E. Ono et al., 1986, for details.)

The entire display was presented through a Polaroid filter, and Polaroid filter glasses were worn by the observers. The display, viewed through a small aperture mounted on the chinrest, was seen by the left eye on half the trials, and by the right eye on the other half. Two Nonius circles (monocularly seen circles) and two vertical fixation bars were presented on the random dot display. One of the Nonius circles was presented with a fixation bar at its bottom and the other with a fixation bar at its top. The Nonius circle with the bottom fixation bar was placed to the left of the Nonius circle with the top fixation bar. Both were presented through Polaroid filters so that the Nonius circle and fixation bar on the left were seen by the right eye and the Nonius circle and fixation bar on the right were seen by the left eye (see Figure 1). The observers "crossed" their eyes so that the Nonius circles appeared aligned and the two bars fused into a single bar. The extent of head movement was restricted to $6 \mathrm{~cm}$, so that observers would keep the two bars fused while moving their heads from side to side. When the two bars were fused, the convergence state was set at a particular convergence condition.

The display was viewed at a distance of $80 \mathrm{~cm}$. The largest extent of dot movement was $0.9 \mathrm{~cm}$ for a $30-\mathrm{cm}$ movement of the chinrest. The shape of the dot (cell) varied as the head moved, and the extent to which it changed depended on how much the dot moved. The largest change occurred midway between the peak and trough. The extent of motion parallax was equivalent to $16.5^{\prime}$ of disparity (see Graham \& Rogers, 1982, for the unit of "equivalent disparity"). Equivalent disparity is derived from the extent of the relative motion of a retinal image, for a $6-\mathrm{cm}$ horizontal head movement, that is analogous to an interocular distance of $6 \mathrm{~cm}$. This disparity corresponded to a peak-to-trough depth of $4.5 \mathrm{~cm}$ at a viewing distance of $80 \mathrm{~cm}$. The visible portion of the screen was $30 \mathrm{~cm}$ wide and $28 \mathrm{~cm}$ high, the peak-to-peak distance of the corrugations was $18 \mathrm{~cm}$, and the random dot density was $8.8 \mathrm{dots} / \mathrm{cm}^{2}$. The dot (cell) size was $2.4 \mathrm{~mm}^{2}$. The fixation bars were $0.5 \mathrm{~cm}$ wide and $2.5 \mathrm{~cm}$ high, and the Nonius circles were $0.4 \mathrm{~cm}$ in diameter. Expressed in degrees of visual angle, the screen was $21.2^{\circ}$ wide and $19.9^{\circ} \mathrm{high}$, the peak-to-peak distance was $12.8^{\circ}$, and there were $17.2 \mathrm{dots} / \mathrm{deg}^{2}$. The dot (cell) size was $10 \mathrm{~mm}^{2}$. The fixation bars were $0.4^{\circ}$ wide and $1.8^{\circ}$ high, and the Nonius circles were $0.3^{\circ}$ in diameter.

In the $60-\mathrm{cm}$ convergence condition, the Nonius circles and the two bars were placed $2 \mathrm{~cm}$ apart so that, when fused, the convergence angles were set for a distance of approximately $60 \mathrm{~cm}$. In the 40-cm convergence condition, they were placed $6 \mathrm{~cm}$ apart so that, when fused, the convergence angles were set for a distance of approximately $40 \mathrm{~cm}$. The values of 40 and $60 \mathrm{~cm}$ are approximate, since there are individual differences in interocular distance. They were computed using an interocular distance of $6 \mathrm{~cm}$. (See Figure 2 for an illustrative example of the overconvergence conditions.) In the 80-cm convergence condition, a fixation circle, superimposed on Nonius bars, was fixed on the center of the screen. The Nonius bars were presented through Polaroid filters such that the top bar was seen by one eye and the bottom bar by the other. The observers looked at the fixation circle such that the convergence angles were set for a distance of $80 \mathrm{~cm}$ (the actual distance

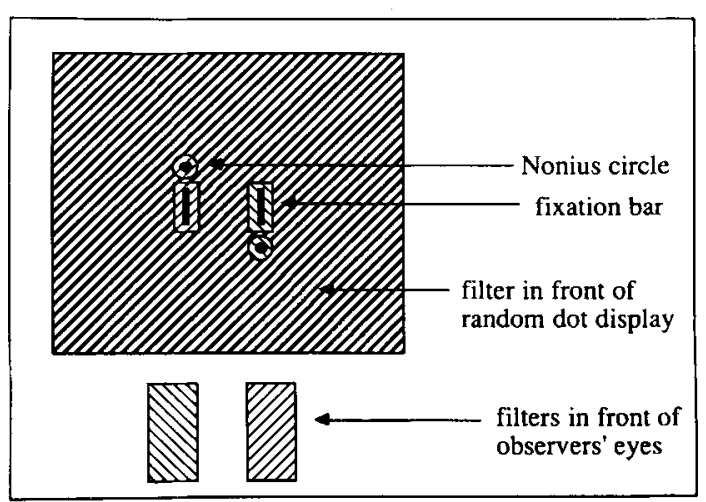

Figure 1. Front view of the Polaroid filter arrangement. The orientation of the shading indicates the orientation of the Polaroid filters. The distance between the two fixation bars varied as the convergence state was varied. (Diagram not to scale.) 


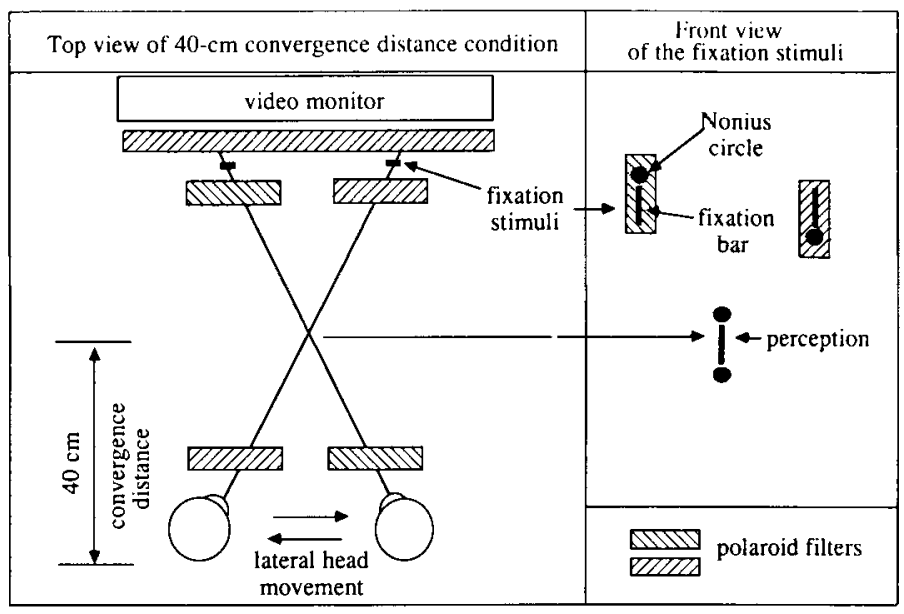

Figure 2. Schematic illustration of the $40-\mathrm{cm}$ convergence distance condition. The orientation of the shading iddicates the orientation of the Polaroid filters. One eye (e.g. left eye) saw the screen, the bottom monocular circle, and the fixation bar at its top. The other eye (e.g., right eye) saw the top monocular circle, and the fixation bar at its bottom. While moving the head from side to side, the observer fused the fixation bars so that the circles looked aligned. (Diagram not to scale.)

of the display). The required accommodation was constant for each convergence condition. The observers were required to maintain alignment of the Nonius targets while moving their heads from side to side, and to manually adjust the spatial separation between two viewed rods to match the apparent depth of the display. They were encouraged to take as much time as was necessary to make their evaluations, and they were permitted to view the rods while they were moving their heads and when they were making their final reports. After the depth reports, they were asked to report whether they saw a "rocking" motion-an apparently rigid three-dimensional surface rotating along a vertical axis.

There were two blocks of 12 trials, with a break after the first block. Within each block, four judgments were made for each of the three convergence conditions. The order of the conditions was randomized within a block for each observer, except in the starting condition, which was counterbalanced among observers.

Twelve observers, recruited from the York University community in Toronto, Canada, participated in Experiment 1. All the observers were naive as to the purpose of the experiment, but 10 of them were experienced in judging depth from a parallactic display. The results of 3 of the observers, who could not align the Nonius circles, were not included in the analysis, since their convergence angles were not set for the distance required. Moreover, another observer always saw a rocking motion with apparent depth. Because apparent motion appears to be at the expense of apparent depth (Gogel, 1980; M. E. Ono et al., 1986), these results were also excluded from the analysis.

\section{Results and Discussion}

To determine whether depth produced by motion parallax is calibrated by convergence, an analysis was performed on the apparent depth from the observers who saw depth without motion for the three convergence conditions $(40,60$, and $80 \mathrm{~cm})$. The data for the analysis were the mean apparent depth values obtained for each observer in each condition. The mean apparent depth values for each condition are shown in Table 1. A one-way repeated measures analysis of variance was performed with ob- servers ( 8 individuals) as a randomized block and convergence $(40,60,80 \mathrm{~cm})$ as a factor.

Although the three mean depths were larger than that expected for the $80-\mathrm{cm}$ convergence condition $(4.5 \mathrm{~cm})$, they were not statistically different among the three convergence conditions $[F(2,14)=0.20, p>.05]$. The larger size of the means is discussed at the end of this section. The lack of difference shows that apparent depth produced by motion parallax does not vary significantly with convergence, when the extent of motion parallax, the actual absolute distance of the display, and the required accommodation are kept constant. These results suggest that motion parallax is not calibrated by convergence. The results are similar to those of Cormack and Menendez (1983), who found that apparent depth produced by retinal disparity does not vary with forced convergence.

Although our results are consistent with those of Cormack and Menendez (1983), they are not consistent with those of Wallach and Zuckerman (1963) or O'Leary and Wallach (1980), who reported that depth perception produced by retinal disparity is contingent upon oculomotor adjustments. One possible explanation is that depth perception is contingent upon convergence only when the apparent distance of the display covaries with convergence: Retinal disparity and motion parallax may be calibrated

Table 1

Mean Apparent Depth Values (and Standard Deviations) for the Three Convergence Distances

\begin{tabular}{cccc}
\hline & \multicolumn{3}{c}{ Convergence Distance } \\
\cline { 2 - 4 } & 40 & 60 & 80 \\
\hline$M$ & 5.6 & 5.7 & 5.3 \\
$S D$ & 3.1 & 2.0 & 1.9 \\
\hline
\end{tabular}

Note-Distances are in centimeters. $n=8$ 
with apparent distance but not with convergence. For example, in Wallach and Zuckerman's (1963) and O'Leary and Wallach's (1980) experiments, manipulation of convergence may have changed the apparent distance of the display, and in Cormack and Menendez's (1983) experiment as well as in our own, it may not have changed it. This interpretation is reasonable in light of data showing that retinal disparity is calibrated with apparent distance. That is, Wallach and Zuckerman's (1963) results showed that apparent distance manipulated by perspective cues calibrates retinal disparity for depth perception. Also, O'Leary and Wallach's (1980) results showed that apparent distance, manipulated in one experiment by perspective cues, and in another experiment by size of a familiar object, calibrates retinal disparity for depth perception. Apparent depth arising from retinal disparity is contingent upon the apparent distance even when the apparent distance is different from the distance at which the oculomotor adjustments are set.

Although apparent distance sometimes changes as a function of the convergence state (see Ebenholtz \& Wolfson, 1975; Gogel, 1961, 1977; Heuer, 1986; Richards, 1979; Richards \& Miller, 1969), the apparent distance of the display in Experiment 1 probably did not. In Experiment 1 , monocular information about the distance of the display, such as accommodation and angular size of the display, was kept constant across conditions, and the display was viewed monocularly. These factors may have contributed to the apparent distance's not changing with the convergence state. Thus, if motion parallax is calibrated by apparent distance but not with the convergence state, apparent depth would remain the same for the different convergence states, when the manipulation of convergence does not change the apparent distance of the display. Furthermore, the larger than expected apparent depths in the three conditions can be explained if the apparent distances of the display are larger than the actual distance. The difficulty with this argument is that we have no direct evidence that apparent distance did not change, or that it was larger; so in Experiment 3, we checked the effect of our manipulation to change the apparent distance.

\section{EXPERIMENT 2}

Experiment 2 was designed to determine whether apparent depth varies with apparent distance. This experiment is analogous to O'Leary and Wallach's (1980) experiment on depth perception as a result of retinal disparity. In their experiment, apparent depth was determined in a display presented at two different apparent distances, which were manipulated by varying the retinal size of a familiar object. In our Experiment 2, observers judged the apparent depth arising from a parallactic display presented at two different apparent distances manipulated in the same way. The display consisted of one portion showing Canadian dollar bills and another showing random dots; it was viewed monocularly at one absolute distance and presented at two different apparent distances.
In one condition, normally sized dollar bills were presented, and the apparent distance was expected to be the same as the actual distance of the display (normal-size condition). In another, the actual size of the dollar bills was reduced, and the display was expected to appear to be farther away than it actually was (70\%-size condition). If motion parallax is calibrated by apparent distance, the apparent depth between the dollar bills and the random dot surfaces should be larger when the apparent distance is greater.

\section{Method}

The same apparatus as in Experiment 1 was used to simulate two surfaces at different distances. A video camera displayed a random dot surface on the bottom half of a TV monitor and a dollar bill surface on its top half. The amplitude of the horizontal deflection signal of the monitor was modified by a square wave signal from a function generator (Wavetek Model 184) in synchrony with the vertical synchronized signal of the monitor. The observer's head movement was yoked to the relative movement of the two different surfaces, so that as the head moved, the entire dollar bill's surface moved in one direction relative to the head movement, while the entire random dot surface moved to the same extent but in the opposite direction relative to the head movement. The part moving in the opposite direction relative to the head movement was seen as being closer than the part moving in the same direction.

The display was $80 \mathrm{~cm}$ from the observers. The chinrest had a range of $30 \mathrm{~cm}$, and the dots moved $0.9 \mathrm{~cm}$ for the full range of movement of the chinrest. The extent of motion parallax was equivalent to $16.5^{\prime}$ of disparity. This disparity corresponded to a depth interval of $4.5 \mathrm{~cm}$ at a viewing distance of $80 \mathrm{~cm}$. The visible portion of the screen was $30 \mathrm{~cm}$ wide and $28 \mathrm{~cm}$ high. The height of each surface was $14 \mathrm{~cm}$. Expressed in degrees of visual angle, the screen was $21.2^{\circ}$ wide and $19.9^{\circ}$ high, and the height of each surface was $10^{\circ}$.

In the normal-size condition, the display consisted of a surface of normally sized dollar bills (linear size of a dollar bill = $15.3 \times 6.9 \mathrm{~cm})$, positioned above a random dot surface with a density of $8.8 \mathrm{dots} / \mathrm{cm}^{2}$. The apparent distance of the display was expected to be about $80 \mathrm{~cm}$ (the actual distance of the display). Expressed in degrees of visual angle, the normally sized dollar bill was $10.9^{\circ}$ wide and $4.9^{\circ} \mathrm{high}$, and there were $17.2 \mathrm{dots} / \mathrm{deg}^{2}$ at a distance of $80 \mathrm{~cm}$. In the $70 \%$-size condition, the display consisted of a surface of $30 \%$-reduced dollar bills (linear size of a reduced dollar bill $=10.7 \times 4.8 \mathrm{~cm}$ ), positioned above a random dot surface with a density increased by $204 \%$. The apparent distance of the display was expected to be about $114 \mathrm{~cm}$. Expressed in degrees of visual angles, the reduced-size dollar bill was $7.7^{\circ}$ wide and $3.4^{\circ}$ high, and there were $35.1 \mathrm{dots} / \mathrm{deg}^{2}$ at a distance of $80 \mathrm{~cm}$.

The observers viewed the stimulus monocularly through a small aperture mounted on the chinrest, so that only the display and a black cardboard surround could be seen. After observing the dollars for a period of $10 \mathrm{sec}$, the observers reported the apparent depth in the same way as in Experiment 1: They adjusted the spatial separation between two rods to match the apparent depth of the display, while moving their heads at a speed and to an extent with which they felt comfortable. Care was taken to ensure that the observers never saw the display while not looking through the small aperture.

There were two blocks of eight trials. For each block, judgments were made for both the $70 \%$-size and the normal-size conditions. Four trials of each condition were included within a block. The order of the conditions was randomized within a block for each observer, except in the starting condition, which was counterbalanced among observers. 
The same 12 observers who participated in Experiment 1 served in Experiment 2. All observers were naive as to the purpose of the experiment, but all observers were experienced with judging depth from a parallactic display.

\section{Results and Discussion}

To determine whether depth produced by motion parallax is calibrated by apparent distance, an analysis was performed on the reported apparent depth for the two apparent distance conditions (70\%-size and normal-size conditions). The data for this analysis were the mean apparent depth values obtained for each observer in each condition. The mean apparent depth values for each condition are shown in Table 2. A $t$ test for matched groups was performed on the data.

The reported apparent depth was greater for the $70 \%$ size condition than for the normal-size condition $[t(11)$ $=2.9, p<.05$ ]. Ten observers out of 12 (sign test, $p<.05)$ reported seeing more depth when viewing the reduced dollar bills than when viewing the normally sized bills. (An interesting fact should be noted: One observer out of the 2 who did not report more apparent depth when the reduced bills were displayed was a Japanese woman on her first visit to Canada, who was not familiar with the size of the Canadian dollar bills.) These results suggest that apparent depth produced by a given extent of motion parallax varies with apparent distance, and, therefore, they suggest that the visual system uses apparent distance to calibrate motion parallax. The results are similar to results found in the processing of depth produced by retinal disparity. As previously discussed in Experiment 1, the results of both O'Leary and Wallach's (1980) and Wallach and Zuckerman's (1963) studies showed that retinal disparity is calibrated by apparent distance to produce depth. Experimental evidence suggests that the visual system uses apparent distance to calibrate both motion parallax and retinal disparity for depth perception.

Although the results were as expected, an alternative interpretation is possible. Gogel (personal communication, December 20, 1986) pointed out that in Experiment 2, as well as in O'Leary and Wallach's (1980) experiment, the manipulation of perceived distance may have been ineffective but still obtained the difference in the reported depth. That is, the observers may have perceived the normally sized and the reduced dollar bills to be at the same distance and based their judgments of depth relative to the size of respective dollar bills. The alternative interpretation states that (1) the reported depth does not

Table 2

Mean Apparent Depth Values (and Standard Deviations) for the Normal-Size and 70\%-Size Conditions

\begin{tabular}{ccc}
\hline & \multicolumn{2}{c}{ Dollar Bill Size } \\
\cline { 2 - 3 } & Normal & $70 \%$ \\
\hline$M$ & 4.0 & 5.1 \\
$S D$ & 1.9 & 2.5 \\
\hline
\end{tabular}

Note-Values are in centimeters. $n=12$. consist of perceived depth but rather of cognitive judgments of depth, (2) the perceived sizes of the dollar bills (normal or reduced) were veridical in both conditions, and (3) the observers reported the magnitude of relative depth to be proportional to the size of the respective dollar bills. For example, consider the perceived depth to be $4 \mathrm{~cm}$ in both conditions, and the dollar bills perceived as $30 \%$ smaller in one condition than in the other. The relative depth may be judged and reported as being $5.2 \mathrm{~cm}$ (30\% larger) when the reduced dollar bills are displayed even if the perceived depth remains $4 \mathrm{~cm}$, because the perceived depth is proportionately larger with respect to the perceived size of the dollar bill. Experiment 3 was designed so that this alternative interpretation would not apply.

\section{EXPERIMENT 3}

Experiment 3 was designed to determine whether apparent depth is contingent upon apparent distance when the apparent distance is manipulated in a different manner than in Experiment 2. A random dot display was presented at a fixed distance in three conditions. In the primary condition (apparently near condition), an aperture was placed in front of the random dot display, and the whole display was moved relative to the head movement so that it appeared in the plane of the aperture. In a comparison condition (moving 120-cm condition), the aperture was removed, and the random dot display was moved as in the apparently near condition. In a second comparison condition (stationary $120-\mathrm{cm}$ condition), the aperture was removed, but the random dot display was stationary. The observers adjusted the extent of motion parallax until the depth appeared to be identical to that of a standard stimulus. Furthermore, when the apparent distance of the random dot display was changed, the apparent distance of the display was measured to ascertain the effective manipulation of the apparent distance. If motion parallax is calibrated according to apparent distance, the extent of motion parallax should be adjusted larger when the display appears closer than its actual distance.

\section{Method}

The display simulated one and a third cycles of a horizontal threedimensional square wave. A video camera sent a pattern of random dots to a video projector, which projected the pattern onto a rear projected screen. The observer's head was supported on a chinrest that could move $10 \mathrm{~cm}$ along a track parallel to the projection screen. A potentiometer yoked the horizontal movement of the observer's head to the horizontal deflection signal of the projector, so that the relative movement of the random dots was synchronized with the head movement. The amplitude of the signal of the projector was modified by a square wave signal from a function generator (NF FG-142) in synchrony with the vertical signal of the projector. The amplitude and the direction of the square wave signal varied with the position of the chinrest, so that the image on the projestion screen was systematically distorted with the observer's head movement producing four adjacent bands of dots. As the observer's head moved, two bands of dots (the first and third) moved in one direction relative to the head movement, while the two others (the 
second and fourth) moved at the same distance but in the opposite direction. The part moving in the opposite direction relative to the head movement was seen as being closer than the part moving in the same direction. The random dot pattern was projected on the projection screen, which was positioned $120 \mathrm{~cm}$ from the observer. The pattern was $18 \mathrm{~cm}$ wide and $18 \mathrm{~cm}$ high, one cycle of the square wave was $14 \mathrm{~cm}$, and the random dot density was 6 dots $/ \mathrm{cm}^{2}$. Expressed in degrees of visual angle, the random dot pattern was $8.58^{\circ}$ wide and $8.58^{\circ}$ high, one cycle of the square wave was $6.68^{\circ}$, and there were 4.35 dots $/ \mathrm{deg}^{2}$ at a distance of $120 \mathrm{~cm}$.

In the primary (apparently near) condition, an induction screen was placed in front of the random dot pattern on the projection screen. The induction screen, made of coarse cardboard, had an aperture $12 \mathrm{~cm}$ wide and $12 \mathrm{~cm}$ high $\left(8.58^{\circ}\right.$ wide and $8.58^{\circ}$ high). The induction screen was placed in front of the projection screen, $80 \mathrm{~cm}$ from the observer. The random dot pattern was visible through the central aperture in the induction screen. It was moved in the direction opposite to the head movement such that it appeared almost stationary in the plane of the induction screen. (See Gogel's [1956] discussion of equal distance tendency.) As the head moved laterally, the entire random dot pattern was moved in the direction opposite to the horizontal head movement, so that the center of the aperture, the center of the random dot pattern, and the viewing eye were always collinear. This was done by generating lateral movement of the video projector as the observer's head moved horizontally. The video projector moved $0.5 \mathrm{~cm}$ laterally for every $1 \mathrm{~cm}$ of head movement. (See Figure 3 for an illustration of the experimental setup.)

There were two comparison control conditions (the moving 120$\mathrm{cm}$ condition and the stationary $120-\mathrm{cm}$ condition). In the moving $120-\mathrm{cm}$ condition, the random dot pattern was presented on the projection screen, but no induction screen was placed in front of it. The pattern was moved laterally in the same way as in the apparently near condition, and it appeared to move at the distance of the projection screen. In the stationary $120-\mathrm{cm}$ condition, the random dot pattern was stationary, presented without an induction screen in front of it. As in the apparently near condition, the random dot pattern appeared stationary but at the distance of the projection screen.

In the three conditions, the observers adjusted the extent of motion parallax by turning a knob that controlled the extent of dot move-

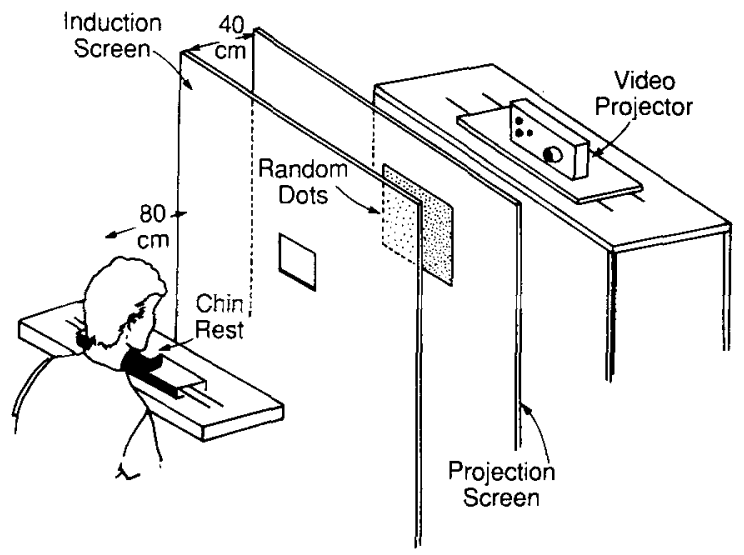

Figure 3. Schematic illustration of the experimental setup used in the apparently near condition. An induction screen was placed in front of the random dot pattern on the projection screen. The random dot pattern was visible through the central aperture in the induction screen. It was moved in the direction opposite to the head movement such that it appeared stationary in the plane of the induction screen. This was done by generating lateral movement of the video projector as the observer's head moved horizontally. ment relative to the head movement. They adjusted the extent of motion parallax until the depth appeared to be $1 \mathrm{~cm}$. The task was to match the apparent depth to a "remembered" 1 -cm depth, and a standard stimulus of $1 \mathrm{~cm}$ depth was shown whenever a subject wished to see it. The standard stimulus consisted of a square wave (1-cm depth) $12 \mathrm{~cm}$ wide and $30 \mathrm{~cm}$ high, the height of one step of the square wave was $4.7 \mathrm{~cm}$, and the random dot density was 6 dots $/ \mathrm{cm}^{2}$. The standard stimulus was presented at $40 \mathrm{~cm}$ from the observer. It was raised to an observer's eye level when requested, and lowered afterward so that it could not be seen while the observer adjusted the extent of motion parallax. The observers viewed the display with their preferred eyes, and they were encouraged to take their time in making their adjustments of the extent of motion parallax.

There were 12 trials for each condition. Six trials of each condition were run successively in a block, so there were two blocks of 6 trials for each condition. The order of the blocks was randomized for each observer, except for the starting block, which was counterbalanced among observers. Six measures of the apparent distance of the random dot pattern were taken after a block of 6 trials of the apparently near condition.

To verify whether the manipulation of apparent distance was effective, two sets of additional data were collected on different days. For the first set, the observers reported the apparent depth between the random dot pattern and the induction screen by matching it to the depth between the experimenter's hand and the induction screen. There were eight trials for each observer. For this measurement, the random dot pattern was presented without relative dot motion. Except for the absence of relative dot motion, the stimulus arrangement was identical to that in the apparently near condition. The display was, on the average, perceived $9.9 \mathrm{~cm}$ behind the induction screen. The manipulation of the induction screen was not totally effective, probably because of a slight mechanical lag of the projector: This lag could have produced the apparent depth between the random dot pattern and the induction screen from dynamic occlusion and motion parallax (see H. Ono, Rogers, Ohmi, \& M. E. Ono, 1988). For the second set, the observers reported the apparent absolute distances of the induction screen at $80 \mathrm{~cm}$, and the projection screen at $120 \mathrm{~cm}$ without the induction screen in front of it at $80 \mathrm{~cm}$. (The projection screen by itself is thought to be an induction screen to see the display at $120 \mathrm{~cm}$.) The apparent distances of each screen were reported eight times by spreading the two hands until the distance between the hands matched the perceived distance. The two means (with standard deviations) of the apparent distance were $85 \mathrm{~cm}(12.49 \mathrm{~cm})$ for the induction screen and $123.9 \mathrm{~cm}(12.11 \mathrm{~cm})$ for the projection screen. The difference between the two means is large enough to mitigate the induction's not being totally effective in the apparently near condition. Therefore, if averaged equivalent disparity is larger in the apparently near condition than in the other two conditions, the conclusion can be made that motion parallax is calibrated with apparent distance.

Six observers recruited from the Tsukuba University community in Japan participated in Experiment 3. All observers were naive as to the purpose of the experiment, but 4 of them were experienced in judging depth from a parallactic display.

\section{Results and Discussion}

To determine whether motion parallax is calibrated by apparent distance to produce depth, an analysis was performed on the adjusted extent of motion parallax transformed in equivalent disparity (in minutes of arc). The data for this analysis are the averaged equivalent disparities for each subject for the apparently near condition, the stationary $120-\mathrm{cm}$ condition, and the moving $120-\mathrm{cm}$ condition. The mean equivalent disparity for each condition is shown in Table 3 . One-way repeated measures 
Table 3

Mean Equivalent Disparity Values (and Standard Deviations) for Three Conditions

Conditions

\begin{tabular}{lccc} 
& Moving 120-cm & Stationary 120-cm & Apparently Near \\
\hline$M$ & 1.43 & 1.37 & 2.46 \\
$S D$ & 0.37 & 0.34 & 0.57 \\
\hline
\end{tabular}

Note-Values are in minutes of arc. $n=6$.

analysis of variance was performed with observers $(6$ individuals) as a randomized block and with condition (moving $120-\mathrm{cm}$, stationary $120-\mathrm{cm}$, and apparently near) as a factor.

There was a significant difference in the averaged equivalent disparity for the three apparent distance conditions $[F(2,10)=74.14, p<.001]$. Tukey tests showed that the averaged equivalent disparity for the apparently near condition was significantly larger than for the sta-, tionary $120-\mathrm{cm}$ condition $[q(2,10)=15.08, p<.001]$ and significantly larger than for the moving $120-\mathrm{cm}$ condition $[q(2,10)=14.29, p<.01]$. The averaged equivalent disparity for the stationary $120-\mathrm{cm}$ condition and for the moving $120-\mathrm{cm}$ condition was not statistically different $[q(2,10)=0.79, p>.05]$. These results show that the required extent of motion parallax varies with apparent distance when the actual absolute distance of the display is kept constant. These results clearly show that motion parallax is calibrated with apparent distance for depth perception. ${ }^{2}$ Our conclusion is similar to that based on the results in the processing of depth produced by retinal disparity. As previously discussed in the sections on Experiments 1 and 2, the results of both O'Leary and Wallach's (1980) and Wallach and Zuckerman's (1963) studies suggest that retinal disparity is calibrated by apparent distance to produce depth.

\section{GENERAL DISCUSSION}

The results of this study show that the visual system uses apparent distance but not convergence as absolute distance information to calibrate motion parallax for depth perception. The results of Experiment 1 show that the visual system does not calibrate motion parallax with the convergence state for depth perception. Although an alternative interpretation could explain the results of Experiment 2, the results of Experiment 3 clearly show that the visual system calibrates motion parallax with apparent distance for depth perception. These results, and those reported in the literature on binocular disparity, suggest a similarity between the processing of motion parallax and the processing of binocular disparity: The visual system calibrates motion parallax as well as retinal disparity with apparent distance for depth perception. Presumably, the source of absolute distance information does not matter; be it convergence, familiar size, or any other cues, the visual system uses the "processed" rather than the original information. Thus, for an absolute distance cue to be a factor, it must first affect the apparent distance. Accord- ingly, depth perception is contingent upon convergence only when convergence changes the apparent distance.

Since apparent distance calibrates motion parallax for depth perception, the visual system has the potential to provide depth constancy solely with monocular information. Depth constancy can occur when the visual system uses a monocular cue for relative depth (i.e., motion parallax) and calibrates it with apparent distance, which can be determined solely by monocular cues of absolute distance. For example, apparent distance can be determined by the extent of eye rotation relative to head movement during fixation, vertical angle of regard, and familiarly sized objects. The fact that motion parallax is calibrated with apparent distance demonstrates that, contrary to common belief, retinal disparity is not necessary for depth constancy. Motion parallax and retinal disparity provide redundant information, since both are used by the visual system to provide depth constancy. Either motion parallax or retinal disparity is sufficient but not necessary for depth constancy.

Although motion parallax and retinal disparity provide redundant information about depth, the range of viewing distances in which depth perception occurs may be larger with motion parallax than with retinal disparity, since the extent of head movement can be larger than the interocular distance. This suggestion contradicts M. E. Ono et al.'s (1986) conclusion that depth constancy with motion parallax is limited to near distances. Their conclusion, however, now appears to be wrong, in light of new results by Nakamizo and Saida (personal communication, January 16,1989 ) which show that the limit is due to the change in the visual field's size rather than to the viewing distances. Thus, the relative contribution of the two sources of information to depth constancy for different distances has yet to be explored.

\section{REFERENCES}

BouRDON, B. (1902). La perception visuelle de l'espace [Visual space perception]. Paris: Librairie Schleincher Frères.

CoRmack, R., MENENDEz, A. (1983). The role of convergence in stereoscopic depth constancy (Tech. Rep. No. N14-0001-83C-0001, Office of Naval Research). Nashville, TN: Vanderbilt University.

Ebenholtz, S. M., \& Wolfson, D. M. (1975). Perceptual aftereffects of sustained convergence. Perception \& Psychophysics, 17, 485-491.

FrIED, A. H. (1974). Convergence as a cue to distance (Doctoral dissertation, New School for Social Research, 1973). Dissertation Abstracts International, 3, 3247B. (University Microfilms No. 74-146)

GoGEL, W. C. (1956). The tendency to see objects as equidistant and its inverse relation to lateral separation. Psychological Monographs, 70(11, Whole No. 418).

GoGEL, W. C. (1961). Convergence as a cue to absolute distance. Journal of Psychology, 52, 287-301.

GoGEL, W. C. (1972). Scalar perceptions with binocular cues of distance. American Journal of Psychology, 85, 477-498.

GoGEL, W. C. (1977). An indirect measure of perceived distance from oculomotor cues. Perception \& Psychophysics, 21, 3-11.

GoGEL, W. C. (1978). Size, distance and depth perception. In E. C. Carterette \& M. P. Friedman (Eds.), Handbook of perception (Vol. 9, pp. 305-308). New York: Academic Press.

GOGEL, W. C. (1980). The sensing of retinal motion. Perception \& Psychophysics, 28, 155-163.

Graham, M. (1983). Motion parallax and the perception of three- 
dimensional surface. Unpublished doctoral dissertation, University of St-Andrews, Scotland.

Graham, M., \& Rogers, B. (1982). Simultaneous and successive contrast effects in the perception of depth from motion-parallax and stereoscopic information. Perception, 11, 247-262.

HeINE, L. (1905). Über Wahrnehmung und Vorstellung von Entferhungsunterschieden [On perception and conception of distance differences]. Albrecht von Graefe's Archiv fur Klinische und Experimentelle Ophthalmologie, 61, 484-498.

HeLmHOLtz, H. voN. (1962). Helmholtz's treatise on physiological optics: Vol. 3 (3rd ed.). (J. P. C. Southall, Ed. \& Trans.). New York: Dover. (Original work published 1910)

Heuer, H. (1986). Perceptual aftereffects of sustained fixation and oculomotor changes. In D. G. Bouwhuis, B. Bridgeman, D. A. Owens, W. L. Shebilske, \& P. Wolf (Eds.), Sensorimotor interactions in space perception and action (pp. 247-261). Amsterdam: Elsevier.

ITTELsON, W. H. (1960). Visual space perception. New York: SpringerVerlag.

NAKAYAMA, K. (1985). Biological image motion processing: A review. Vision Research, 25, 625-660.

O'LeARY, A., \& Wallach, H. (1980). Familiar size and linear perspective as distance cues in stereoscopic depth constancy. Perception \& Psychophysics, 27, 131-135.

ONO, H. (1970). Some thoughts on different perceptual tasks related to size and distance. In J. C. Baird (Ed.), Human space perception: Proceedings of the Dartmouth Conference. Psychonomic Monograph Supplements, 3(13, Whole No. 45), 143-151.

ONo, H., Comerford, J. (1977). Stereoscopic depth constancy. In W. Epstein (Ed.), Perception stability and constancy in visual perception: Mechanism and process (pp. 91-128). New York: WileyInterscience.

Ono, H., Rogers, B. J., OhMi, M., * ONo, M. E. (1988). Dynamic occlusion and motion parallax in depth perception. Perception, 17, 255-266.

ONo, M. E., Rivest, J., \& ONo, H. (1986). Depth perception as a function of motion parallax and absolute-distance information. Journal of Experimental Psychology: Human Perception \& Performance, 12, 331-337.

RICHARDS, W. (1979). Size-distance transformations. In Pattern recognition in biological and technical systems (pp. 276-287). New York: Springer-Verlag.

Richards, W., \& Miller, J. F., JR. (1969). Convergence as a cue to depth. Perception \& Psychoph)|sics, 5, 317-320.
RoGERS, B. (1986). The perception of surface curvature from disparity and motion parallax cues. Investigative Ophthalmology \& Visual Science, 27(ARVO Suppl. 3), 181.

Rogers, B., \& Graham, M. (1979). Motion parallax as an independent cue for depth perception. Perception, 8, 125-134.

Wallach, H., Gillam, B., \& CaRDillo, L. (1979). Some consequences of stereoscopic depth constancy. Perception \& Psychophysics, 26, 235-240.

Wallach, H., \& Zuckerman, C. (1963). The constancy of stereoscopic depth. American Journal of Psychology, 76, 404-412.

\section{NOTES}

1. The term stereoscopic depth constancy is usually used for depth constancy with retinal disparity. Because stereo means solid in Greek, it can therefore also apply to depth constancy with motion parallax. To avoid confusion, it is not used in this paper.

2. Braunstein (personal communication, January 30, 1989) pointed out that "viewing through an aperture occurred only in the apparently near condition" and that "any effects of viewing through an aperture were thus not controlled." We could not control for this effect completely, because we were unable to move the aperture of the induction screen back and forth at $120 \mathrm{~cm}$, but a supplementary experiment with three conditions was conducted. The three conditions were: without aperture (no induction screen in front of the projection screen), with the same angular sized aperture $(18 \times 18 \mathrm{~cm}$ : same size as the size of the retinal projection of the screen used in the apparently near condition), and with the same linear sized aperture $(12 \times 12 \mathrm{~cm}$ : same size as the distal size of the screen used in the apparently near condition). The procedure was the same as in the primary experiment. The means (with standard deviations) of the adjusted equivalent disparities in minutes of arc were: $1.36(0.32), 1.39(0.35)$, and $1.42(0.31)$, respectively. One-way repeated measures analysis of variance with observers (6 individuals) as a randomized block and with condition (moving $120-\mathrm{cm}$, stationary 120-cm, and apparently near) as a factor indicated that there were no statistical differences among the three conditions $[\mathrm{F}(2,10)=1.62$, n.s.]. The results show that there is no effect of viewing through an aperture at $120 \mathrm{~cm}$, and they suggest that viewing through aperture per se is not the determining factor for the larger adjusted equivalent disparity found in the apparently near condition.

(Manuscript received November 14, 1988; revision accepted for publication April 26, 1989.) 